\title{
CRITICALITY OF SWITCHING CLASSES OF REVERSIBLE 2-STRUCTURES LABELED BY AN ABELIAN GROUP
}

\author{
Houmem BeLKhechine \\ University of Carthage \\ Institut Préparatoire aux Études d'Ingénieurs de Bizerte, BP 64 \\ 7021 Bizerte, Tunisie \\ e-mail: houmem@gmail.com \\ Pierre Ille \\ Institut de Mathématiques de Marseille \\ CNRS UMR 7373, 13453 Marseille, France \\ e-mail: pierre.ille@univ-amu.fr
}

AND

ROBERT E. WOODROW

Department of Mathematics and Statistics University of Calgary, 2500 University Drive

Calgary, Alberta, Canada T2N 1N4

e-mail: woodrow@ucalgary.ca

\begin{abstract}
Let $V$ be a finite vertex set and let $(\mathbb{A},+)$ be a finite abelian group. An $\mathbb{A}$-labeled and reversible 2-structure defined on $V$ is a function $g:(V \times V) \backslash$ $\{(v, v): v \in V\} \longrightarrow \mathbb{A}$ such that for distinct $u, v \in V, g(u, v)=-g(v, u)$. The set of $\mathbb{A}$-labeled and reversible 2-structures defined on $V$ is denoted by $\mathscr{L}(V, \mathbb{A})$. Given $g \in \mathscr{L}(V, \mathbb{A})$, a subset $X$ of $V$ is a clan of $g$ if for any $x, y \in X$ and $v \in V \backslash X, g(x, v)=g(y, v)$. For example, $\emptyset, V$ and $\{v\}$ (for $v \in V$ ) are clans of $g$, called trivial. An element $g$ of $\mathscr{L}(V, \mathbb{A})$ is primitive if $|V| \geq 3$ and all the clans of $g$ are trivial.

The set of the functions from $V$ to $\mathbb{A}$ is denoted by $\mathscr{S}(V, \mathbb{A})$. Given $g \in \mathscr{L}(V, \mathbb{A})$, with each $s \in \mathscr{S}(V, \mathbb{A})$ is associated the switch $g^{s}$ of $g$ by $s$ defined as follows: given distinct $x, y \in V, g^{s}(x, y)=s(x)+g(x, y)-s(y)$. The switching class of $g$ is $\left\{g^{s}: s \in \mathscr{S}(V, \mathbb{A})\right\}$. Given a switching class $\mathfrak{S} \subseteq \mathscr{L}(V, \mathbb{A})$ and $X \subseteq V,\left\{g_{\uparrow(X \times X) \backslash\{(x, x): x \in X\}}: g \in \mathfrak{S}\right\}$ is a switching class, denoted by $\mathfrak{S}[X]$.
\end{abstract}


Given a switching class $\mathfrak{S} \subseteq \mathscr{L}(V, \mathbb{A})$, a subset $X$ of $V$ is a clan of $\mathfrak{S}$ if $X$ is a clan of some $g \in \mathfrak{S}$. For instance, every $X \subseteq V$ such that $\min (|X|, \mid V \backslash$ $X \mid) \leq 1$ is a clan of $\mathfrak{S}$, called trivial. A switching class $\mathfrak{S} \subseteq \mathscr{L}(V, \mathbb{A})$ is primitive if $|V| \geq 4$ and all the clans of $\mathfrak{S}$ are trivial. Given a primitive switching class $\mathfrak{S} \subseteq \mathscr{L}(V, \mathbb{A}), \mathfrak{S}$ is critical if for each $v \in V, \mathfrak{S}-v$ is not primitive. First, we translate the main results on the primitivity of $\mathbb{A}$-labeled and reversible 2 -structures in terms of switching classes. For instance, we prove the following. For a primitive switching class $\mathfrak{S} \subseteq \mathscr{L}(V, \mathbb{A})$ such that $|V| \geq 8$, there exist $u, v \in V$ such that $u \neq v$ and $\mathfrak{S}[V \backslash\{u, v\}]$ is primitive. Second, we characterize the critical switching classes by using some of the critical digraphs described in [Y. Boudabous and P. Ille, Indecomposability graph and critical vertices of an indecomposable graph, Discrete Math. 309 (2009) 2839-2846].

Keywords: labeled and reversible 2-structure, switching class, clan, primitivity, criticality.

2010 Mathematics Subject Classification: 05C75.

\section{REFERENCES}

[1] P. Bonizzoni, Primitive 2-structures with the $(n-2)$-property, Theoret. Comput. Sci. 132 (1994) 151-178. doi:10.1016/0304-3975(94)90231-3

[2] Y. Boudabous and P. Ille, Indecomposability graph and critical vertices of an indecomposable graph, Discrete Math. 309 (2009) 2839-2846. doi:10.1016/j.disc.2008.07.015

[3] Y. Cheng and A.L. Wells, Switching classes of directed graphs, J. Combin. Theory Ser. B 40 (1986) 169-186. doi:10.1016/0095-8956(86)90075-4

[4] A. Cournier and P. Ille, Minimal indecomposable graph, Discrete Math. 183 (1998) 61-80. doi:10.1016/S0012-365X(97)00077-0

[5] A. Ehrenfeucht, T. Harju and G. Rozenberg, The Theory of 2-Structures, A Framework for Decomposition and Transformation of Graphs (World Scientific, 1999). doi:10.1142/4197

[6] A. Ehrenfeucht and G. Rozenberg, Theory of 2-structures, Part I: clans, basic subclasses, and morphisms, Theoret. Comput. Sci. 70 (1990) 277-303. doi:10.1016/0304-3975(90)90129-6

[7] A. Ehrenfeucht and G. Rozenberg, Primitivity is hereditary for 2-structures, Theoret. Comput. Sci. 70 (1990) 343-358.

[8] P. Ille, Recognition problem in reconstruction for decomposable relations, in: Finite and Infinite Combinatorics in Sets and Logic, B. Sands, N. Sauer and R. Woodrow (Ed(s)), (Kluwer Academic Publishers, 1993) 189-198.

doi:10.1007/978-94-011-2080-7_13 
[9] P. Ille, Indecomposable graphs, Discrete Math. 173 (1997) 71-78. doi:10.1016/S0012-365X(96)00097-0

[10] P. Ille and R. Woodrow, Decomposition tree of a lexicographic product of binary structures, Discrete Math. 311 (2011) 2346-2358. doi:10.1016/j.disc.2011.05.037

[11] J.H. Schmerl and W.T. Trotter, Critically indecomposable partially ordered sets, graphs, tournaments and other binary relational structures, Discrete Math. 113 (1993) 191-205.

doi:10.1016/0012-365X(93)90516-V

[12] J.J. Seidel, Strongly regular graphs of L2 type and of triangular type, in: Proc. Kon. Nederl. Akad. Wetensch. Ser. A (Indag. Math. 29, 1967) 188-196.

doi:10.1016/S1385-7258(67)50031-8

Received 27 September 2015

Revised 29 March 2016

Accepted 29 March 2016 